Brit. Heart f., 1969, 31, 711.

\title{
Acute Haemodynamic Effects of Frusemide in Patients with Normal and Raised Left Atrial Pressures
}

\author{
S. LAL, J. G. MURTAGH*, A. M. POLLOCK, E. FLETCHER, AND P. F. BINNION \\ From the Cardiovascular Unit, Belfast City Hospital, Belfast, $N$. Ireland
}

Frusemide is a well-established diuretic (Vorburger, 1964; McKenzie, Fairley, and Baird, 1966; Kirkendall and Stein, 1968), and a mild hypotensive when given in a dosage of over $120 \mathrm{mg}$. daily (Davidov, Kakaviatos, and Finnerty, 1967). It is commonly used in the treatment of acute pulmonary oedema with beneficial results (Stason et al., 1966; Peltola, 1965; Biagi and Bapat, 1967). Because of the obvious difficulties, its haemodynamic effects in acute pulmonary oedema have seldom been studied and the stress has been on its diuretic action and clinical improvement after its administration. It appeared to us that a study of the haemodynamic action of the drug on left atrial pressure in selected patients would be helpful in elucidating the mechanism of improvement of the cardiac status.

Though some work has been done on the systemic haemodynamic effects of frusemide (International Furosemide Symposium, Bad Homburg, 1963), little or no information is available on its effect on left atrial pressure and pulmonary haemodynamics in patients either with normal or raised left atrial pressure. It was because of this paucity of information in association with its obvious clinical benefit in patients with acute pulmonary oedema that this study was instituted to investigate its haemodynamic effects in these 2 groups of patients.

\section{Patients and Methods}

A total of 23 patients was studied, and for convenience the patients were divided into 2 groups. In the first group were 8 patients whose mean left atrial pressure was under $10 \mathrm{~mm}$. Hg. In the second group 15 patients had mitral valve disease with a mean left atrial pressure above $10 \mathrm{~mm}$. $\mathrm{Hg}$, six of them had episodes of paroxysmal nocturnal dyspnoea, and all experienced dyspnoea on exertion.

All patients had right heart catheterization and either

Received May 9, 1969.

$\star$ In receipt of a Research Award from the British Heart a brachial artery needle or retrograde aortic catheter inserted. In addition, those with valvular heart disease had left atrial catheterization performed using a modified Brockenbrough needle with a blunt-ended stylette. Cardiac outputs were measured by injecting indocyanine green dye $(2.5 \mathrm{mg}$.) into the pulmonary artery with constant arterial withdrawal through a Gilford densitometer, and the dye curve was recorded on either a Sanborn photographic recorder or an ultraviolet recorder.

Pressures in the pulmonary artery, brachial artery or central aorta, and the left atrium were measured at rest under mild sedation using a Sanborn pressure transducer (Model 267B) coupled to a Sanborn photographic recorder. The reference point for all pressure determinations was $5 \mathrm{~cm}$. below the sternal angle and a simultaneous electrocardiogram was taken with all pressure measurements. One or two control cardiac outputs were determined and their average taken. Frusemide was injected directly into the pulmonary artery through the catheter in a dose of $\mathbf{4 0} \mathrm{mg}$. over a two-minute period, and the above parameters were measured 15 minutes and $\mathbf{4 5}$ minutes later.

The following values were derived from the results:

Total pulmonary vascular resistance (dynes./sec./cm.-5)

$$
=\frac{\text { Mean pulmonary artery pressure }(\mathrm{mm} . \mathrm{Hg})}{\text { Cardiac output }(\mathrm{ml} . / \mathrm{sec} .)} \times 1332
$$

Pulmonary vascular resistance

(dynes./sec./cm. ${ }^{-5}$ )

$$
=\frac{\begin{array}{l}
\text { Mean pulmonary artery pressure - } \\
\text { Mean left atrial pressure }(\mathrm{mm} . \mathrm{Hg})
\end{array}}{\text { Cardiac output }(\mathrm{ml} . / \mathrm{sec} .)} \times 1332
$$

Total systemic resistance (dynes./sec. $/ \mathrm{cm}^{-5}$ )

$$
=\frac{\begin{array}{c}
\text { Mean aortic or brachial artery pressure } \\
(\mathrm{mm} . \mathrm{Hg})
\end{array}}{\text { Cardiac output }(\mathrm{ml} . / \mathrm{sec} .)} \times 1332
$$

Central blood volume (ml.)

= Appearance time of dye (sec.) $\times$ cardiac output $(\mathrm{ml} . / \mathrm{sec}$.)

Pressure-time index

(mm. Hg sec./min.)

$=$ Ejection time $(\mathrm{sec}$.$) \times mean ejection pressure (\mathrm{mm} . \mathrm{Hg}) \times$ heart rate (beats/min.) 
where mean ejection pressure was taken electronically from a catheter in the ascending aorta and the index was taken as an estimate of left ventricular work.

\section{RESULTS}

Patients with Mean Left Atrial Pressures

$$
<10 \mathrm{~mm} \text {. } \mathrm{Hg}
$$

Pulmonary Artery, Left Atrial, and Systemic Arterial Pressures. There were no significant changes in these parameters either 15 or 45 minutes after intravenous frusemide (Table I).

Cardiac Output. There was an average fall of 13.6 per cent after 15 minutes and 24.6 per cent after 45 minutes (Table I).

Calculated Resistances. Though the frusemide caused no change in total pulmonary or pulmonary vascular resistance, there was an average rise of 26.6 per cent and 32.6 per cent in total systemic resistance 15 and 45 minutes after frusemide (Table I).

\section{Patients with Mean Left Atrial Pressures $>10 \mathrm{~mm}$. $\mathrm{Hg}$}

Pulmonary Artery. The reduction in mean pulmonary artery pressure was only significant 45 minutes after frusemide (mean $=-9.0 \mathrm{~mm}$. $\mathrm{Hg}$, S.E. $= \pm 1 \cdot 5)$ (Table II).
Left Atrium. Though the average fall in left atrial pressure was small 15 and 45 minutes after frusemide (mean $=-3.0$ and $-4.5 \mathrm{~mm}$. $\mathrm{Hg}$, respectively), these changes were significantly different from control though not from one another (Table II).

Cardiac Output. Fifteen minutes after frusemide the changes in cardiac output were variable (mean $=+0.8 \%$, S.E. \pm 3.2 ) and not significant, while after 45 minutes cardiac output was reduced by $17 \cdot 4$ per cent which was highly significantly different from both the control and 15-minute cardiac output measurements (Table II).

Total Systemic Resistance. Though there was no change in mean systemic arterial pressure produced by frusemide, after 45 minutes there was an increase of $20 \cdot 8$ per cent (S.E. $\pm 5.3 \%$ ) in total systemic resistance which is significantly different from control $(0.01<\mathrm{p}<0.001)$ and the 15 -minute measurement $(0.05<\mathrm{p}<0.02)$ (Table II).

Pulmonary Resistance. There was a significant fall in total pulmonary resistance after 15 minutes (mean $=-14.0 \%$ ) and 45 minutes after the intravenous frusemide (mean $=-16.4 \%$ ) though the $45-$ minute fall was not greater than the 15-minute fall ( $p>0.05)$, but there were no significant changes in pulmonary vascular resistance (Table II).

TABLE I

HAEMODYNAMIC DATA FROM PATIENTS WITH

\begin{tabular}{|c|c|c|c|c|c|c|c|}
\hline \multicolumn{3}{|c|}{$\begin{array}{l}\text { Case No., sex, and } \\
\text { age (yr.) }\end{array}$} & \multirow{2}{*}{$\begin{array}{c}\text { Rhythm } \\
\text { Sinus }\end{array}$} & \multirow{2}{*}{$\begin{array}{c}\text { Clinical diagnosis } \\
\text { Chronic bronchitis }\end{array}$} & \multirow{2}{*}{$\begin{array}{l}\text { Observation } \\
\text { Control } \\
\text { After } 15 \mathrm{~min} \text {. } \\
\text { After } 45 \mathrm{~min} .\end{array}$} & \multirow{2}{*}{$\begin{array}{c}\begin{array}{c}\text { Mean pulmonary } \\
\text { artery pressure } \\
\text { (mm. Hg) }\end{array} \\
13 \\
16 \\
15\end{array}$} & \multirow{2}{*}{$\begin{array}{c}\begin{array}{c}\text { Mean systemic } \\
\text { artery pressure } \\
\text { (mm. Hg) }\end{array} \\
- \\
\overline{111}\end{array}$} \\
\hline 1 & $\mathbf{M}$ & 53 & & & & & \\
\hline 2 & $\mathbf{F}$ & 45 & ” & Chronic bronchitis & $\begin{array}{l}\text { Control } \\
\text { After } 15 \mathrm{~min} . \\
\text { After } 45 \mathrm{~min} .\end{array}$ & $\begin{array}{l}14 \\
14 \\
11\end{array}$ & $\begin{array}{l}82 \\
84 \\
80\end{array}$ \\
\hline 3 & $M$ & 17 & " & $\begin{array}{l}\text { "Innocent systolic } \\
\text { murmur" }\end{array}$ & $\begin{array}{l}\text { Control } \\
\text { After } 15 \mathrm{~min} \text {. } \\
\text { After } 45 \mathrm{~min} .\end{array}$ & $\frac{11}{16}$ & $\begin{array}{l}104 \\
114 \\
104\end{array}$ \\
\hline 4 & $\mathbf{F}$ & 39 & , & $\begin{array}{l}\text { Mild mitral } \\
\text { incompetence }\end{array}$ & $\begin{array}{l}\text { Control } \\
\text { After } 15 \mathrm{~min} \text {. } \\
\text { After } 45 \mathrm{~min} \text {. }\end{array}$ & $\begin{array}{l}15 \\
14 \\
12\end{array}$ & $\begin{array}{l}85 \\
98 \\
81\end{array}$ \\
\hline 5 & $\mathbf{F}$ & 25 & 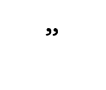 & Mild aortic stenosis & $\begin{array}{l}\text { Control } \\
\text { After } 15 \mathrm{~min} \text {. } \\
\text { After } 45 \mathrm{~min} \text {. }\end{array}$ & E & $\begin{array}{l}79 \\
65 \\
63\end{array}$ \\
\hline 6 & $\mathbf{F}$ & 43 & ” & Mild mitral stenosis & $\begin{array}{l}\text { Control } \\
\text { After } 15 \mathrm{~min} \text {. } \\
\text { After } 45 \mathrm{~min} .\end{array}$ & $\begin{array}{l}15 \\
14 \\
13\end{array}$ & $\begin{array}{l}66 \\
65 \\
63\end{array}$ \\
\hline 7 & $\mathbf{F}$ & 23 & " & Mild mitral stenosis & $\begin{array}{l}\text { Control } \\
\text { After } 15 \mathrm{~min} \text {. } \\
\text { After } 45 \mathrm{~min} \text {. }\end{array}$ & $\begin{array}{r}18 \\
14 \\
9\end{array}$ & $\begin{array}{l}78 \\
85 \\
70\end{array}$ \\
\hline 8 & $\mathbf{F}$ & 44 & $"$ & Mild mitral stenosis & $\begin{array}{l}\text { Control } \\
\text { After } 15 \mathrm{~min} \text {. } \\
\text { After } 45 \mathrm{~min} \text {. }\end{array}$ & $\begin{array}{r}13 \\
10 \\
8\end{array}$ & $\begin{array}{l}67 \\
73 \\
69\end{array}$ \\
\hline
\end{tabular}


Table III summarizes the significance of the above results.

In addition to the above measurements the following data were obtained.

Central Blood Volume. In 3 patients (Cases 13, 22 , and 23) changes in central blood volume were calculated and after 15 minutes the average fall was 10.0 per cent, and 17.2 per cent 45 minutes after the frusemide had been given.

Pressure-time Index (Table IV). In a few patients the pressure-time was calculated and used as a measure of left ventricular function. While there was no change after 15 minutes, there was an average fall of 16.4 per cent 45 minutes after frusemide.

\section{Discussion}

In patients with mean left atrial pressures less than $10 \mathrm{~mm}$. $\mathrm{Hg}$ the essential haemodynamic change produced by intravenous frusemide was a conspicuous reduction in cardiac output. This reduction in cardiac output was accompanied by an increase in total systemic resistance which maintained mean systemic arterial pressure, presumably due to the activity of baroreceptor reflexes. This reduction in cardiac output was due to a reduced stroke volume and the underlying mechanism responsible for this is probably a reduction in plasma volume (Wolfer and Schneider, 1963), though measurements of this were not performed in the above study.

Many of the patients with mitral valve disease where the left atrial mean pressure exceeded $10 \mathrm{~mm}$. $\mathrm{Hg}$ had experienced paroxysmal nocturnal dyspnoea which, like other forms of pulmonary oedema, probably occurs when the mean left atrial pressure exceeds a value of about $30 \mathrm{~mm}$. $\mathrm{Hg}$. This is consistent with Starling's law of capillaries and has been noted at cardiac catheterization in patients with mitral stenosis (personal observations). All patients with raised left atrial pressures and mitral stenosis had experienced progressive dyspnoea on exertion. It was because of these facts that they were considered suitable alternatives to patients with acute pulmonary oedema, and the effect of a powerful diuretic on their haemodynamics should reflect the changes expected when the beneficial action of frusemide is noted in patients with acute pulmonary oedema (Peltola, 1965; Stason et al., 1966).

When the mean left atrial pressure was above 10 $\mathrm{mm} . \mathrm{Hg}$, intravenous frusemide reduced the cardiac output 45 minutes later and the total systemic arterial resistance increased, just as in the case of the other group of patients with normal mean left atrial pressure. In addition, however, the former group reduced their mean left atrial and mean pulmonary arterial pressures, so that the strain on

MEAN LEFT ATRIAL PRESSURES $<10 \mathrm{~mm}$. Hg

\begin{tabular}{|c|c|c|c|c|c|}
\hline $\begin{array}{l}\text { Mean left } \\
\text { atrial pressure } \\
(\mathrm{mm} . \mathrm{Hg})\end{array}$ & $\begin{array}{l}\text { Mean gradient } \\
\text { across pulmonary } \\
\text { vascular tree } \\
(\mathrm{mm} . \mathrm{Hg})\end{array}$ & $\underset{(1 . / \min .)}{\text { Cardiac output }}$ & $\begin{array}{l}\text { Total pulmonary } \\
\text { vascular resistance } \\
{\text { (dynes/sec. } / \mathrm{cm}^{-5} \text { ) }}^{\text {dyne }}\end{array}$ & 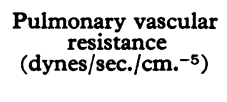 & 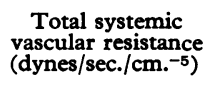 \\
\hline $\begin{array}{l}4 \\
4 \\
4\end{array}$ & $\begin{array}{r}9 \\
12 \\
11\end{array}$ & $\begin{array}{l}3 \cdot 24 \\
3 \cdot 68 \\
3 \cdot 23\end{array}$ & $\begin{array}{l}321 \\
347 \\
371\end{array}$ & $\begin{array}{l}222 \\
260 \\
272\end{array}$ & $\overline{2744}$ \\
\hline E & - & $\begin{array}{l}2 \cdot 86 \\
2 \cdot 35 \\
2 \cdot 19\end{array}$ & $\begin{array}{l}392 \\
476 \\
402\end{array}$ & 二 & $\begin{array}{l}2295 \\
2850 \\
2922\end{array}$ \\
\hline $\begin{array}{l}5 \\
7 \\
5\end{array}$ & $\frac{6}{11}$ & $\begin{array}{l}6 \cdot 15 \\
5 \cdot 81 \\
4 \cdot 18\end{array}$ & $\frac{143}{306}$ & $\frac{65}{211}$ & $\begin{array}{l}1351 \\
1569 \\
1990\end{array}$ \\
\hline $\begin{array}{l}7 \\
6 \\
3\end{array}$ & $\begin{array}{l}8 \\
8 \\
9\end{array}$ & $\begin{array}{l}3 \cdot 69 \\
2 \cdot 73 \\
2 \cdot 74\end{array}$ & $\begin{array}{r}326 \\
410 \\
350\end{array}$ & $\begin{array}{l}174 \\
234 \\
263\end{array}$ & $\begin{array}{l}1841 \\
2869 \\
2363\end{array}$ \\
\hline $\begin{array}{l}9 \\
4 \\
3\end{array}$ & E & $\begin{array}{l}4 \cdot 92 \\
3 \cdot 15 \\
2 \cdot 56\end{array}$ & 二 & E & $\begin{array}{l}1283 \\
1647 \\
1969\end{array}$ \\
\hline $\begin{array}{r}10 \\
9 \\
7\end{array}$ & $\begin{array}{l}5 \\
5 \\
6\end{array}$ & $\begin{array}{l}5 \cdot 19 \\
4 \cdot 14 \\
3 \cdot 77\end{array}$ & $\begin{array}{l}231 \\
270 \\
276\end{array}$ & $\begin{array}{r}77 \\
96 \\
127\end{array}$ & $\begin{array}{l}1016 \\
1254 \\
1336\end{array}$ \\
\hline $\begin{array}{r}10 \\
9 \\
5\end{array}$ & $\begin{array}{l}8 \\
5 \\
4\end{array}$ & $\begin{array}{l}5 \cdot 21 \\
4 \cdot 64 \\
3 \cdot 97\end{array}$ & $\begin{array}{l}276 \\
241 \\
181\end{array}$ & $\begin{array}{r}123 \\
86 \\
81\end{array}$ & $\begin{array}{l}1197 \\
1463 \\
1409\end{array}$ \\
\hline $\begin{array}{l}3 \\
4 \\
5\end{array}$ & $\begin{array}{r}10 \\
6 \\
3\end{array}$ & $\begin{array}{l}7 \cdot 92 \\
7 \cdot 45 \\
6 \cdot 65\end{array}$ & $\begin{array}{r}131 \\
107 \\
96\end{array}$ & $\begin{array}{r}101 \\
64 \\
36\end{array}$ & $\begin{array}{l}676 \\
784 \\
830\end{array}$ \\
\hline
\end{tabular}


TABLE II

HAEMODYNAMIC DATA IN PATIENTS WITH

\begin{tabular}{|c|c|c|c|c|c|c|c|}
\hline \multicolumn{3}{|c|}{$\begin{array}{l}\text { Case No., sex, and } \\
\text { age (yr.) }\end{array}$} & \multirow[t]{2}{*}{ Rhythm } & \multirow{2}{*}{\begin{tabular}{|l} 
Clinical diagnosis \\
Mitral stenosis
\end{tabular}} & \multirow{2}{*}{$\begin{array}{l}\text { Observation } \\
\text { Control } \\
\text { After } 15 \mathrm{~min} . \\
\text { After } 45 \mathrm{~min} .\end{array}$} & \multirow{2}{*}{$\begin{array}{c}\begin{array}{c}\text { Mean pulmonary } \\
\text { artery pressure } \\
\text { (mm. Hg) }\end{array} \\
20 \\
15 \\
11\end{array}$} & \multirow{2}{*}{$\begin{array}{c}\begin{array}{c}\text { Mean systemic } \\
\text { artery pressure } \\
\text { (mm. } \mathbf{H g})\end{array} \\
52 \\
50 \\
50\end{array}$} \\
\hline 9 & $\mathbf{F}$ & 32 & & & & & \\
\hline 10 & $\mathbf{F}$ & 38 & و & Mitral stenosis & $\begin{array}{l}\text { Control } \\
\text { After } 15 \mathrm{~min} .\end{array}$ & $\begin{array}{l}20 \\
20\end{array}$ & $\begin{array}{l}101 \\
103\end{array}$ \\
\hline 11 & F & 52 & Atrial fibrillation & Mitral stenosis & $\begin{array}{l}\text { Control } \\
\text { After } 15 \mathrm{~min} \text {. } \\
\text { After } 45 \mathrm{~min} \text {. }\end{array}$ & $\begin{array}{l}30 \\
22 \\
24\end{array}$ & $\begin{array}{l}-\overline{59} \\
63\end{array}$ \\
\hline 12 & F & 61 & " & Mitral stenosis & $\begin{array}{l}\text { Control } \\
\text { After } 15 \mathrm{~min} .\end{array}$ & $\begin{array}{l}32 \\
28\end{array}$ & $\begin{array}{l}124 \\
151\end{array}$ \\
\hline 13 & $\mathbf{F}$ & 57 & Sinus & Mitral stenosis & $\begin{array}{l}\text { Control } \\
\text { After } 15 \mathrm{~min} \text {. } \\
\text { After } 45 \mathrm{~min} \text {. }\end{array}$ & $\begin{array}{l}25 \\
23 \\
19\end{array}$ & $\begin{array}{l}85 \\
85 \\
80\end{array}$ \\
\hline 14 & $\mathbf{F}$ & 49 & Atrial fibrillation & $\begin{array}{l}\text { Mitral stenosis + aortic } \\
\text { stenosis }\end{array}$ & $\begin{array}{l}\text { Control } \\
\text { After } 15 \mathrm{~min} \text {. } \\
\text { After } 45 \mathrm{~min} \text {. }\end{array}$ & $\begin{array}{l}37 \\
40 \\
35\end{array}$ & $\begin{array}{l}74 \\
73 \\
74\end{array}$ \\
\hline 15 & F & 32 & Sinus & Mitral stenosis & $\begin{array}{l}\text { Control } \\
\text { After } 15 \mathrm{~min} \text {. } \\
\text { After } 45 \mathrm{~min} \text {. }\end{array}$ & $\begin{array}{l}35 \\
26 \\
18\end{array}$ & $\begin{array}{l}78 \\
97 \\
75\end{array}$ \\
\hline 16 & $\mathbf{F}$ & 37 & Atrial fibrillation & Mitral stenosis & $\begin{array}{l}\text { Control } \\
\text { After } 15 \mathrm{~min} .\end{array}$ & $\begin{array}{l}42 \\
45\end{array}$ & $\begin{array}{l}77 \\
78\end{array}$ \\
\hline 17 & $\mathbf{F}$ & 55 & Sinus & Mitral stenosis & $\begin{array}{l}\text { Control } \\
\text { After } 15 \mathrm{~min} \text {. } \\
\text { After } 45 \mathrm{~min} \text {. }\end{array}$ & $\begin{array}{l}33 \\
27 \\
27\end{array}$ & $\begin{array}{l}65 \\
65 \\
84\end{array}$ \\
\hline 18 & $\mathbf{F}$ & 30 & و & Mitral stenosis & $\begin{array}{l}\text { Control } \\
\text { After } 15 \mathrm{~min} \text {. } \\
\text { After } 45 \mathrm{~min} \text {. }\end{array}$ & $\begin{array}{l}36 \\
24 \\
23\end{array}$ & $\begin{array}{l}83 \\
75 \\
76\end{array}$ \\
\hline 19 & F & 39 & , & $\begin{array}{l}\text { Mitral stenosis + aortic } \\
\text { insufficiency }\end{array}$ & $\begin{array}{l}\text { Control } \\
\text { After } 15 \mathrm{~min} \text {. } \\
\text { After } 45 \mathrm{~min} \text {. }\end{array}$ & $\begin{array}{l}69 \\
70 \\
66\end{array}$ & $\begin{array}{l}65 \\
73 \\
78\end{array}$ \\
\hline 20 & $\mathbf{F}$ & 51 & Atrial fibrillation & $\begin{array}{l}\text { Mitral stenosis + aortic } \\
\text { insufficiency }\end{array}$ & $\begin{array}{l}\text { Control } \\
\text { After } 15 \mathrm{~min} \text {. } \\
\text { After } 45 \mathrm{~min} \text {. }\end{array}$ & $\begin{array}{l}37 \\
25 \\
23\end{array}$ & $\begin{array}{l}80 \\
64 \\
71\end{array}$ \\
\hline 21 & $\mathbf{M}$ & 44 & " & Mitral stenosis & $\begin{array}{l}\text { Control } \\
\text { After } 15 \mathrm{~min} .\end{array}$ & $\begin{array}{l}51 \\
51\end{array}$ & $\begin{array}{l}83 \\
81\end{array}$ \\
\hline 22 & $\mathbf{F}$ & 41 & Sinus & $\begin{array}{l}\text { Mitral stenosis + aortic } \\
\text { insufficiency }\end{array}$ & $\begin{array}{l}\text { Control } \\
\text { After } 15 \mathrm{~min} \text {. } \\
\text { After } 45 \mathrm{~min} \text {. }\end{array}$ & $\begin{array}{l}27 \\
20 \\
12\end{array}$ & $\begin{array}{l}80 \\
76 \\
56\end{array}$ \\
\hline 23 & $\mathbf{M}$ & 43 & Atrial fibrillation & $\begin{array}{l}\text { Mitral stenosis + mitral } \\
\text { insufficiency }\end{array}$ & $\begin{array}{l}\text { Control } \\
\text { After } 15 \mathrm{~min} \text {. } \\
\text { After } 45 \mathrm{~min} \text {. }\end{array}$ & $\begin{array}{l}36 \\
36 \\
28\end{array}$ & $\begin{array}{l}67 \\
72 \\
73\end{array}$ \\
\hline
\end{tabular}

the right ventricle was smaller and the back pressure on the lung vessels was reduced. It is possible that the latter would reduce the work of ventilation, and in the few patients studied there was in fact a fall in central blood volume, which probably accounts for the reduced dyspnoea to be seen in patients with high left atrial pressures after frusemide therapy (Peltola, 1965; Stason et al., 1966). This is consistent with the work of McCredie (1967) who measured pulmonary extravascular fluid volume using a double isotope technique and showed that this volume was increased when the mean left atrial pressure exceeded $12 \mathrm{~mm} . \mathrm{Hg}$. Reduction of left atrial mean pressure by frusemide would diminish this fluid volume, reduce the work of ventilation, and hence diminish the degree of dyspnoea.

Beside the reduction in the work of the right ventricle due to a lowered mean pulmonary artery pressure, the work of the left ventricle was reduced as measured by the fall in the pressure-time index. Hence, cardiac work, both left and right ventricular, is reduced after intravenous frusemide, and this is in agreement with the work of Rowe et al. (1962) using intravenous chlorothiazide in hypertensive patients. Undoubtedly this is a cause of the clinical improvement seen in patients on diuretic therapy.

Though there was a reduction in pulmonary artery pressure and cardiac output which led to a fall in total pulmonary resistance in patients with 
MEAN LEFT ATRIAL PRESSURE > $10 \mathrm{~mm}$. $\mathrm{Hg}$

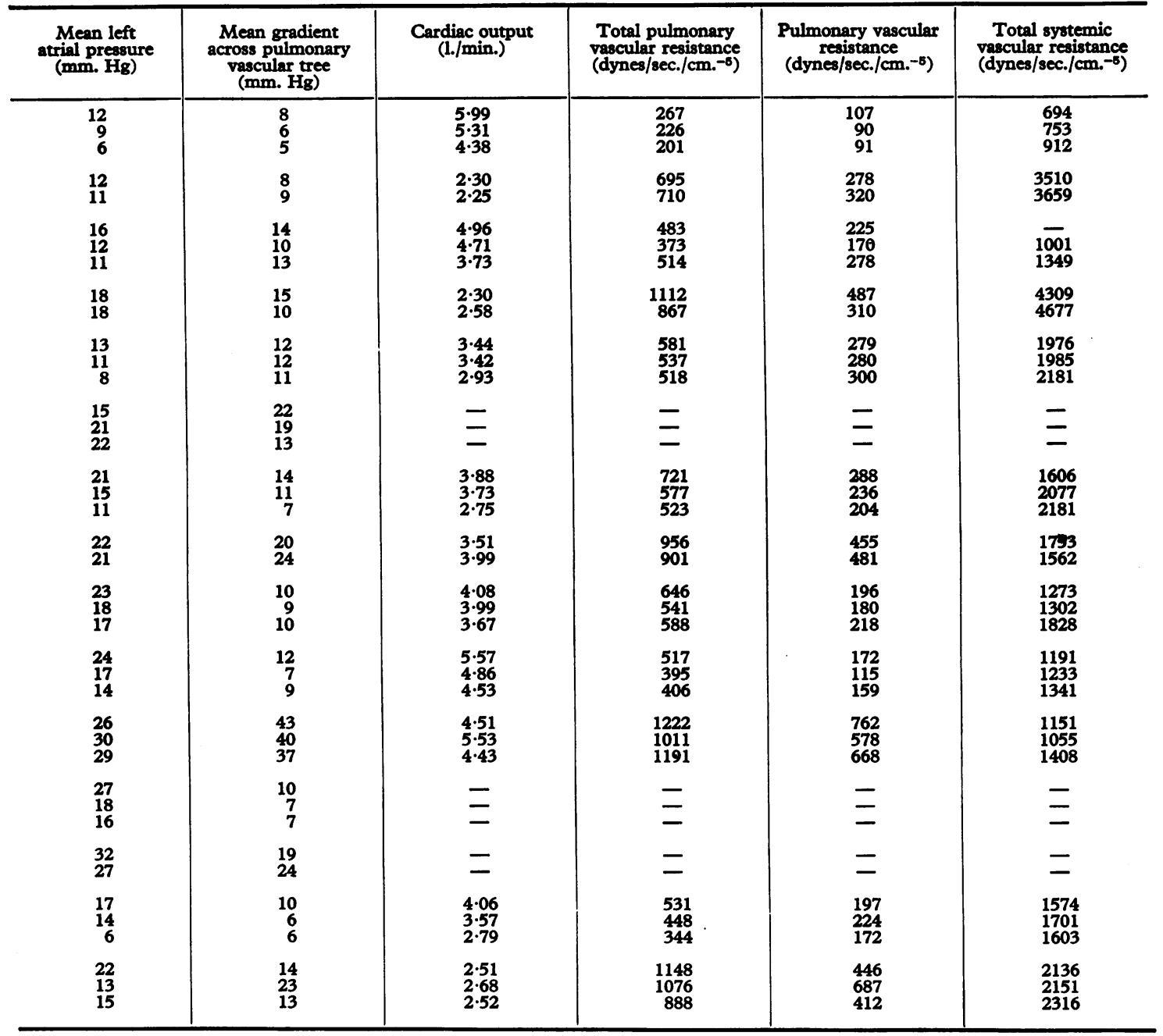

TABLE III

SUMMARY OF HAEMODYNAMIC CHANGES PRODUCED BY INTRAVENOUS FRUSEMIDE

\begin{tabular}{|c|c|c|c|c|}
\hline & \multicolumn{2}{|c|}{ Left atrial pressure ( $<10 \mathrm{~mm} . \mathbf{H g})$} & \multicolumn{2}{|c|}{$\begin{array}{l}\text { Raised left atrial pressure } \\
\text { (> } 10 \mathrm{~mm} . \mathrm{Hg})\end{array}$} \\
\hline & After $15 \mathrm{~min}$. & After $45 \mathrm{~min}$ & After $15 \mathrm{~min}$. & After $45 \mathrm{~min}$. \\
\hline $\begin{array}{l}\text { Mean pulmonary artery pressure }(\Delta) \\
\text { Mean left atrial pressure }(\Delta) \\
\text { Mean systemic arterial pressure }(\Delta) \\
\text { Cardiac output }(\Delta \%) \\
\text { Total pulmonary resistance }(\Delta \%) \\
\text { Pulmonary vascular resistance }(\Delta \%) \\
\text { Total systemic resistance }(\Delta \%)\end{array}$ & $\begin{array}{r}-1 \cdot 0 \\
-0 \cdot 7 \\
+3 \cdot 3 \\
-13 \cdot 6^{\star} \\
+6 \cdot 9 \\
+1 \cdot 9 \\
+26 \cdot 6^{\star}\end{array}$ & $\begin{array}{l}-2 \cdot 1 \\
-2 \cdot 3 \\
-4 \cdot 4 \\
-24 \cdot 6 \star \\
+14 \cdot 0 \\
+44 \cdot 1 \\
+32 \cdot 6 \star\end{array}$ & $\begin{array}{l}-3 \cdot 6 \\
-3 \cdot 0^{\star} \\
+2 \cdot 1 \\
+0 \cdot 8 \\
-14 \cdot 0 \star \\
-6 \cdot 2 \\
+6 \cdot 2\end{array}$ & $\begin{array}{l}-9 \cdot 0^{\star} \\
-4 \cdot 5^{\star} \\
-1 \cdot 5 \\
-17 \cdot 4^{\star} \\
-16 \cdot 4^{\star} \\
-4 \cdot 7 \\
+20 \cdot 8^{\star}\end{array}$ \\
\hline
\end{tabular}

$\Delta$ and $\Delta \%$ are, respectively, the absolute and percentage changes from control values. 
TABLE IV

PRESSURE-TIME INDEX

\begin{tabular}{|c|c|c|c|c|c|c|c|c|c|c|c|}
\hline \multirow{2}{*}{$\begin{array}{l}\text { Case } \\
\text { No. }\end{array}$} & \multirow[t]{2}{*}{$\begin{array}{l}\text { Age } \\
\text { (yr.) }\end{array}$} & \multirow[t]{2}{*}{ Diagnosis } & \multicolumn{3}{|c|}{$\begin{array}{l}\text { Left atrial pressure } \\
(\mathrm{mm} . \mathbf{H g})\end{array}$} & \multicolumn{3}{|c|}{$\begin{array}{c}\text { Mean central aortic } \\
\text { pressure } \\
(\mathbf{m m} . \mathbf{H g})\end{array}$} & \multicolumn{3}{|c|}{$\begin{array}{l}\text { Pressure-time index } \\
\text { (mm. } \mathrm{Hg} \mathrm{sec} / \mathrm{min} .)\end{array}$} \\
\hline & & & *Control & 15 & 45 & Control & 15 & 45 & Control & 15 & 45 \\
\hline $\begin{array}{r}4 \\
13 \\
22 \\
10 \\
+17 \\
+24\end{array}$ & $\begin{array}{l}39 \\
57 \\
41 \\
38 \\
55 \\
56\end{array}$ & $\begin{array}{l}\text { Mild mitral incompetence } \\
\text { Mitral stenosis } \\
\text { Mitral stenosis + aortic insufficiency } \\
\text { Mitral stenosis } \\
\text { Mitral stenosis } \\
\text { ? Cardiomyopathy }\end{array}$ & $\begin{array}{r}7 \\
13 \\
17 \\
12 \\
23 \\
5\end{array}$ & $\begin{array}{r}6 \\
11 \\
14 \\
11 \\
18 \\
5\end{array}$ & $\begin{array}{r}3 \\
8 \\
6 \\
17 \\
4\end{array}$ & $\begin{array}{r}85 \\
85 \\
80 \\
101 \\
65 \\
70\end{array}$ & $\begin{array}{r}98 \\
85 \\
76 \\
103 \\
65 \\
65\end{array}$ & $\begin{array}{l}81 \\
80 \\
56 \\
84 \\
65\end{array}$ & $\begin{array}{l}1824 \\
2111 \\
2125 \\
2234 \\
1474 \\
1053\end{array}$ & $\begin{array}{l}2058 \\
2066 \\
2052 \\
2253 \\
1602 \\
1017\end{array}$ & $\begin{array}{l}1685 \\
1851 \\
1505 \\
2095 \\
1015\end{array}$ \\
\hline
\end{tabular}

* Control = control value before frusemide $40 \mathrm{mg}$. intravenously. $15=15 \mathrm{~min}$. after frusemide injection; $45=45 \mathrm{~min}$. after frusemide injection.

t On daily oral frusemide.

raised left atrial pressures, there was no change in the true resistance of the pulmonary vascular bed (pulmonary vascular resistance), which was not unexpected as this vascular bed is resistant to most pharmacological agents (Harris and Heath, 1962).

Recently, Stampfer et al. (1968) have studied the action of diuretics on exercise tolerance in patients with impaired cardiac function. They noted a fall in mean left atrial and mean pulmonary artery pressures after a diuresis with a fall in cardiac output and ventricular stroke work. In the two studies mentioned above (Rowe et al., 1962; Stampfer et al., 1968) and the results in this paper there was a reduction in cardiac output with diuretic therapy. However, Rader et al. (1964) calculated that there was an increase in cardiac output in patients with congestive heart failure treated with a mercurial diuretic though there was some variability in their results. The above data in patients with raised mean left atrial pressures support strongly the hypothesis that symptoms of impaired cardiac function are due to circulatory congestion and reduction of this relieves the symptoms. Rader et al. (1964) also point out that this impairment occurs whether "cardiac function is improved towards the normal or not", i.e. cardiac output may or may not increase with clinical improvement in congestive heart failure.

Effective therapy either by intravenous digoxin or venesection improved cardiac function in congestive failure by reducing right atrial pressure to cause an increase in cardiac output (McMichael and Sharpey-Schafer, 1944; Howarth, McMichael, and Sharpey-Schafer, 1946). The main conclusion from this work was that cardiac output increased in response to treatment of heart failure. Others usually noted an increase in cardiac output with venesection in patients with congestive heart failure, though this was not consistent (Judson et al., 1955). The emphasis in previous work on the treatment of heart failure has been on an increase in cardiac out- put as congestive heart failure is treated, and the explanation given was that the patient's myocardium was functioning on the descending limb of the Starling curve.

However, the results of our investigations, together with those of Stampfer et al. (1968), are at variance with this hypothesis for there is a decrease in cardiac output, not an increase, with diuretic therapy in heart disease. The increased exercise tolerance seen after treatment in patients with significant mitral stenosis, or other causes of impaired cardiac function (Stampfer et al., 1968) is due, not to an increase in resting cardiac output produced by diuretic therapy, but rather to a reduction in the burden imposed on the right and left ventricles and also on the work load of ventilation.

\section{SUMMARY}

The haemodynamic actions of frusemide $(40 \mathrm{mg}$.) were studied 15 and 45 minutes after intravenous injection in patients with normal or raised left atrial pressures. Where the mean left atrial pressure was less than $10 \mathrm{~mm}$. $\mathrm{Hg}$, the major haemodynamic change was a conspicuous fall in cardiac output due to a reduction in stroke volume with a compensatory increase in calculated systemic resistance considered to be due to baroreceptor reflexes.

When the mean left atrial pressure was increased, the cardiac output was again reduced 45 minutes after intravenous frusemide and total systemic vascular resistance increased. In addition, there was a significant reduction in mean left atrial and mean pulmonary artery pressures such that total pulmonary vascular resistance decreased, though there was no change in the resistance across the pulmonary vascular tree. In addition, a decrease in central blood volume occurred in the 4 patients studied.

Reduction in cardiac output and pulmonary artery pressure in the patients with increased left atrial pressure would reduce the work of the right ven- 
tricle. There was a decrease in left ventricular work (as measured by a pressure-time index), and the reduction in central blood volume would be expected to diminish the work of ventilation.

It is considered that improvement in symptoms in patients with raised left atrial pressure which follows frusemide therapy is due to a reduction in myocardial work load and the work of ventilation, and there is strong evidence against a previous hypothesis that improvement in heart failure is accompanied by an increase in cardiac output because the patient's myocardium had previously been working on the descending limb of a Starling curve.

We wish to thank Dr. Patricia Morton for permission to study patients under her care.

\section{REFERENCES}

Biagi, R. W., and Bapat, B. N. (1967). Frusemide in acute pulmonary oedema. Lancet, 1, 849.

Davidov, M., Kakaviatos, N., and Finnerty, F. A. (1967). Anti-hypertensive properties of furosemide. Circulation, 36, 125.

Harris, P., and Heath, D. (1962). The effect of drugs. In The Human Pulmonary Circulation, pp. 113-126. E. \& S. Livingstone, Edinburgh.

Howarth, S., McMichael, J., and Sharpey-Schafer, E. P. (1946). Effects of venesection in low output heart failure. Clin. Sci., 6, 41.

International Furosemide Symposium, Bad Homburg (1963). Farbwerke Hoechst A.G., Frankfurt.
Judson, W. E., Hollander, W., Hatcher, J. D., Halperin, M. H., and Friedman, I. H. (1955). The cardiohemodynamic effects of venous congestion of the legs or of phlebotomy in patients with and without congestive heart failure. $\mathcal{F}$. clin. Invest., 34, 614 .

Kirkendall, W. M., and Stein, J. H. (1968). Clinical pharmacology of furosemide and ethacrynic acid. Amer. $\mathcal{F}$. Cardiol., 22, 162.

McCredie, M. (1967). Measurement of pulmonary edema in valvular heart disease. Circulation, 36, 381.

McKenzie, I. F. C., Fairley, K. F., and Baird, C. W. (1966). A clinical trial of frusemide ("Lasix"). Med. F. Aust., 1, 879.

McMichael, J., and Sharpey-Schafer, E. P. (1944). The action of intravenous digoxin in man. Quart. F. Med., n.s., 13, 123.

Peltola, P. (1965). Furosemide (Lasix) as a diuretic. Acta med. scand., 177, 777.

Rader, B., Smith, W. W., Berger, A. R., and Eichna, L. W. (1964). Comparison of the hemodynamic effects of mercurial diuretics and digitalis in congestive heart failure. Circulation, 29, 328.

Rowe, G. G., Castillo, C. A., Crosley, A. P., Maxwell, G. M., and Crumpton, C. W. (1962). Acute systemic and coronary hemodynamic effects of chlorothiazide in subjects with systemic arterial hypertension. Amer. F. Cardiol., 10, 183.

Stampfer, M., Epstein, S. E., Beiser, G. D., and Braunwald, E. (1968). Hemodynamic effects of diuresis at rest and during intense upright exercise in patients with impaired cardiac function. Circulation, 37, 900 .

Stason, W. B., Cannon, P. J., Heinemann, H. O., and Laragh, J. H. (1966). Furosemide. A clinical evaluation of its diuretic action. Circulation, 34, 910.

Vorburger, C. (1964). Un nouveau diurétique, le Fursemid. Rev. méd. Suisse rom., 84, 277.

Wolfer, H. J., and Schneider, W. (1963). In International Furosemide Symposium. Farbwerke Hoechst A.G., Frankfurt. 